\title{
The Significance of Ophthalmological Symptoms in Idiopathic Blepharospasm
}

\author{
J. S. ELSTON,${ }^{1}$ C. D. MARSDEN,${ }^{2}$ F. GRANDAS,${ }^{2}$ N. P. QUINN, ${ }^{2}$ \\ London
}

\section{Summary:}

Two hundred and seventy-two patients with idiopathic blepharospasm were reviewed to establish the role of local eye disease in their illness. The majority of patients $(57 \%)$ had symptoms at the onset of their illness such as dryness of the eyes, grittiness, irritation or photophobia suggesting eye lid or ocular surface disease. Detailed ophthalmological examination at the time of presentation had been carried out in 170 of the 272 cases; $64 \%$ of these patients had ocular symptoms, and $40 \%$ had demonstrable ocular surface or eye lid pathology. Such pathology was usually bilateral, chronic and resistant to local treatment. Blepharospasm developed in these patients after a long latent period, often of many years. Unilateral pathology was acute, normally responded well to local treatment, but was followed by the development of bilateral blepharospasm usually within six months. Amongst all 272 patients, those without ocular symptoms at presentation rarely developed them subsequently; if they did, there were no abnormal signs.

The data suggest that ophthalmological disorders may trigger idiopathic blepharospasm in a substantial proportion of cases predisposed to develop this condition.

Idiopathic blepharospasm begins insidiously in the 5 th -7 th decade with an awareness of an increased frequency of blinking or difficulty keeping the eyes open. It usually evolves over a 1 to 2 year period into forceful involuntary spasms of eye closure, which seriously interfere with visual function. Subsequently the spasms may extend into the lower face, jaw and even the neck. The cause is not known, but there is considerable evidence to suggest that it is a form of focal dystonia probably due to organic dysfunction of the basal ganglia. ${ }^{1.2}$

Most cases of idiopathic blepharospasm present to ophthalmologists, since the initial symptoms of the condition - photophobia, discomfort, irritation or dryness of the eyes with frequent blinking - suggest local ocular or periocular pathology. In this paper, we investigate the significance of these symptoms; the data suggest that local eye disease commonly precedes the onset of blepharospasm and may be a significant trigger to the condition.

\section{Patients and Methods:}

Two hundred and seventy-two patients, visually incapacitated by by idiopathic blepharospasm, were investigated. There were 176 women and 96 men (female to male ratio 1.8:1), with an average age at presentation of 55.8 years (range $11-81$ ). One hundred and ninety-four also had lower facial or oro-mandibular dystonia, and 66 had torticollis or

(From ${ }^{1}$ The Department of Clinical Ophthalmology, Moorfields Eye Hospital, and ${ }^{2}$ University Department of Neurology, Institute of Psychiatry, London, UK).

Presented at the Annual Meeting of the Ophthalmological Society of the United Kingdom, April 1988

Correspondence to: Mr. J. S. Elston, The National Hospital for Nervous Diseases, Queen Square, London. WC1N 3BG 
other neck spasms. Fourteen patients (5\%), who presented at an average of 41.8 years, had first degree relatives with blepharospasm.

The original presenting symptoms were elicited and tabulated as either ophthalmological or neurological (Table I). Ophthalmological symptoms were irritation of the eyes or eyelids, dry eyes, grittiness or foreign body sensation and photophobia. Neurological symptoms were increased frequency or awareness of blinking, closure or drooping of the eyelids, or spasms of eye closure. Amongst these 272 cases were 170 patients who had been seen by ophthalmologists at the time these symptoms developed, with slit lamp examination of the eyelids, tear film, conjunctiva, cornea and anterior segments, and measurement of the intraocular pressure.

\section{Results:}

Ophthalmological complaints were present at the onset in $154(57 \%)$ of the 272 patients with blepharospasm (Table I). One hundred and ninety-six $(72 \%)$ of the patients had neurological symptoms from the onset, in the form of increasing frequency or awareness of blinking in $38 \%$, and closing of the upper lid, spasm of eye closure or other movements in $34 \%$ (Table I). Many patients therefore had both ophthalmological and neurological symptoms, but $76(28 \%)$ had only ophthalmological symptoms at the start of their illness. With time, a progression towards the development of spasms of eye closure occurred in all cases. Amongst the 170 patients with a documented ophthalmological examination, ocular presenting symptoms occurred in $109(64 \%)$. In 40 cases there were symptoms but no signs. In 12 of these patients, blepharospasm was evident at the original presentation and it developed over the next 2 years in all but one of the other 28 cases. In the remaining 69 patients $(40 \%)$ including 11 of the 14 with first degree relatives with blepharospasm, significant signs of ophthalmological disease accompanied the symptoms and preceded the onset of blepharospasm.

Unilateral eye disease (19 cases) was less common than bilateral (50 cases see Table
Table I Presenting symptoms of 272 patients with blepharospasm: Symptoms in the 170 cases who had been examined ophthalmologically at the onset of their symptoms are shown in parentheses

\begin{tabular}{lrr}
\hline Symptoms & Number & Percentage \\
\hline A- Ophthalmological & & \\
$\quad$ Irritation & & \\
$\quad$ Dryness & $154(109)$ & $57(64)$ \\
$\quad$ Grittiness & & \\
$\quad$ Photophobia & & \\
B - Neurological & & \\
$\quad$ Increased blinking & $104(89)$ & $38(52)$ \\
$\quad$ Spasms of eye closure & $92(56)$ & $34(33)$ \\
\hline
\end{tabular}

Table II Ophthalmological abnormalities and response to treatment in 69 patients who developed blepharospasm. All patients had appropriate local and/or systemic treatment for their children

Persistent

Ocular condition Number ocular signs

UNILATERAL: 19 cases

Herpes zoster

(ophthalmic)

Herpes simplex

Actinomycosis (lacrimal)

Corneal abrasion

Glaucoma surgery

Enucleation (pain)

Cataract surgery

Penetrating injury

Cryotherapy

Meibomian cyst

BILATERAL: 50 cases

Blepharitis

Contact lens abrasion

Keratocojunctivitis sicca

Dysthyroid

Fuch's dystrophy

Trichiasis surgery

II). In 13 of the 19 , the signs were abolished by appropriate treatment, and in 4 of the remaining 6 with persistent abnormal signs, they were improved. The symptoms were unaffected however and 16 patients had developed blepharospasm within six months.

The other 3 began blinking excessively within a year of their original complaint and had blepharospasm within 2 years. (Fig.1A). 
In all cases the eye spasms were initially worse on the originally affected side. The majority (50 cases) had bilateral eye disease. Treatment had little effect on the signs, which persisted in all cases. Increased blinking developed within six months in 34 cases, within the first year in 46 and by 2 years in all these patients. Blepharospasm took substantially longer to develop and although it was evident within the first six months in 12 cases in the majority it took over 2 years, the average being 3.7 years (Fig.1B). In order to interpret the significance of local eye disease at the onset, or prior to the appearance of

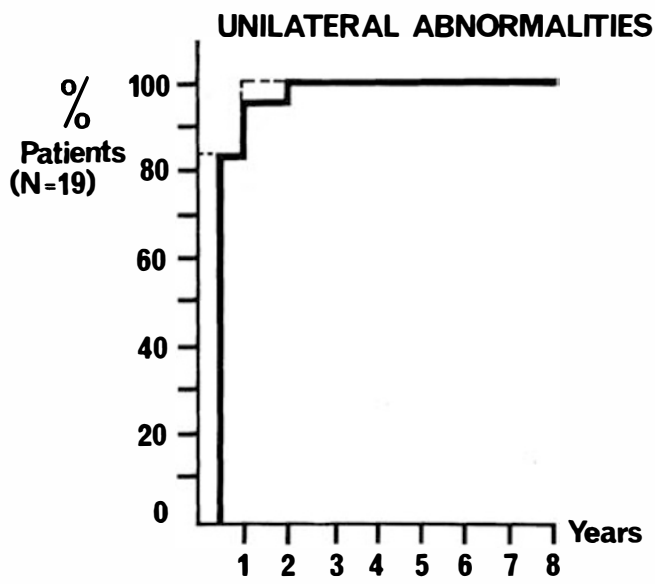

BILATERAL ABNORMALITIES

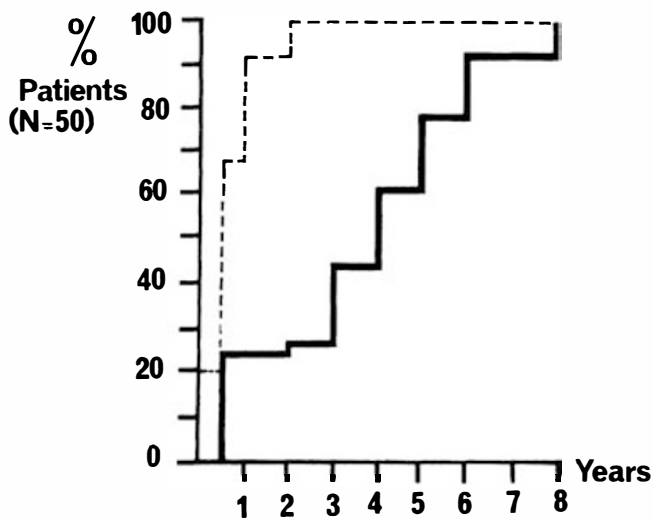

Fig. 1. The temporal relationship between the development of excessive blinking (dashed lines) and blepharospasm (solid lines) in 69 patients whose disease was preceded by A) Unilateral and B) Bilateral ocular abnormalities. blepharospasm we also examined how frequently those with established blepharospasm developed local eye conditions in the course of their illness. Amongst all 272 patients, 118 presented with neurological symptoms alone, but of these only $15(13 \%)$ subsequently developed ocular symptoms (irritation, dryness, grittiness or photophobia) and none developed any abnormal ocular physical signs.

\section{Case Reports:}

Case 1. Unilateral Pathology.

An 84 year old woman developed a central retinal vein occlusion in her left eye which was followed by thrombotic glaucoma; the eye became blind and painful and was removed. The pain however persisted and after two months, increased frequency of blinking was noticed, first in the left lids and then spreading to involve the right lids. The blinking worsened over the next three months and evolved into spasms of eye closure, compromising the vision of the right eye. The patient also has persistent pain in the left socket (Fig.2).

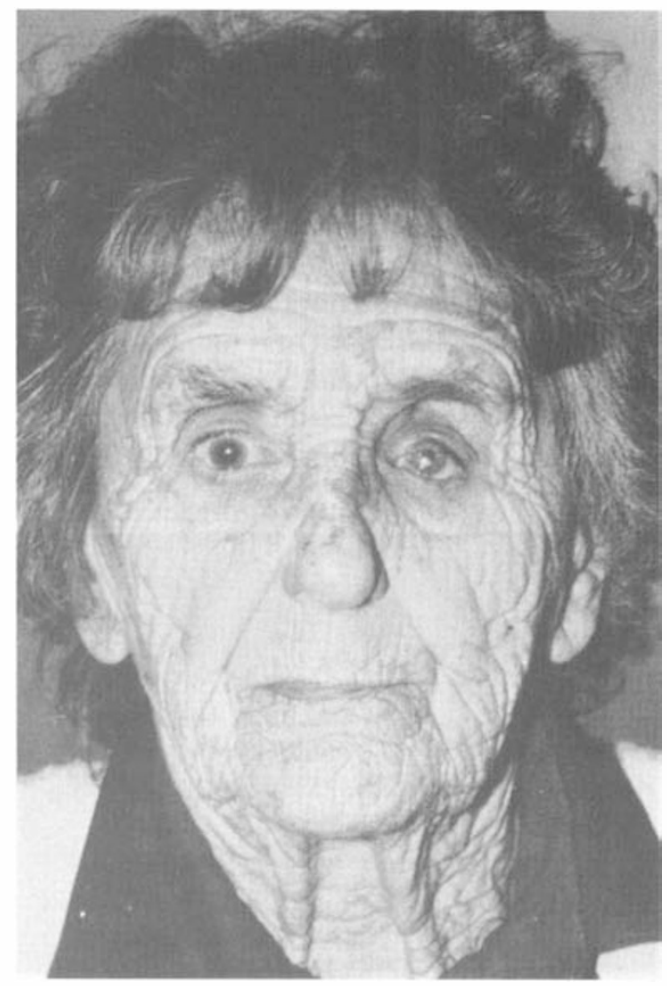

Fig. 2: Case 1. Left prothesis: blepharospasm developed in this patient following the removal of a blind painful eye. 
Case 2. Bilateral Pathology.

A 38 year old woman developed redness, stickiness and crustiness of her eyelid margins with hyperaemia and increased frequency of blinking. Staphyloccal blepharitis was diagnosed and confirmed microbiologically, but local treatment was only partially successful and the condition became chronic (see Fig.3). After three years, the increased frequency of blinking began to progress to spasms of eye closure associated with oro-mandibular dystonia and led to functional blindness.

\section{Discussion:}

The onset of idiopathic blepharospasm is characteristically insidious and may only be recognised in retrospect when the symptoms have progressed to interfere with normal daily activities. Of the 135 patients reviewed by Henderson ${ }^{3}$ the first symptom was usually abnormal blinking, although $28 \%$ had photophobia without ocular abnormality. Similarly, $78 \%$ of the 250 patients analysed by questionnaire by Jankovic and Orman had eye irritation and excessive blinking before they developed blepharospasm. ${ }^{1}$. In spite of this, ocular abnormalities have been noted only rarely in these patients and ophthalmic treatment has been reported to be unhelpful. Ocular symptoms have therefore been regarded as misleading in blepharospasm.

In the 272 patients we have studied, ophthalmological complaints were the most common presenting symptom complex, especially in the 170 patients for whom past ophthalmological documentation was available. Amongst the latter 40 had ocular symptoms with no abnormal signs. In this

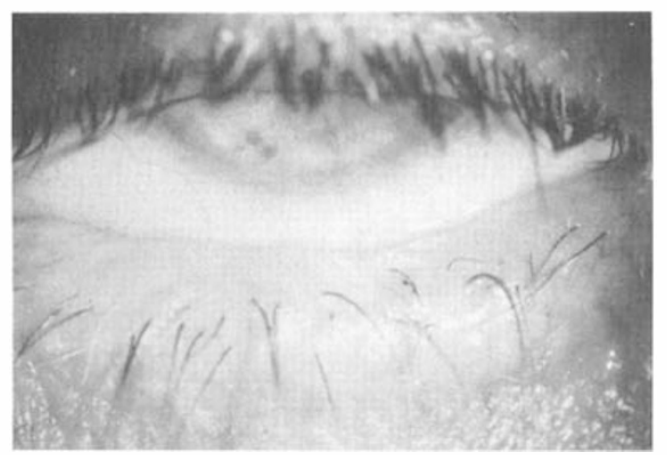

Fig. 3: Case 2. Chronic staphylococcal lid disease. The lid margin is irregular and the peripheral cornea vascularised. group ocular symptoms appeared to accompany the early stages of the natural history of the disease with no overt evidence of ocular surface or lid abnormalities. Such symptoms may possibly be secondary to the centrally determined abnormality of the blink reflex thought to be responsible for blepharospasm. ${ }^{4}$ However, only $13 \%$ of the patients whose blepharospasm began with neurological complaints developed ocular symptoms subsequently, and abnormal ocular signs never developed. Accordingly, the significance of the high incidence of ocular symptoms without signs at presentation is uncertain; it could indicate that transient local eye conditions, at the onset, may have been the trigger to the subsequent development of blepharospasm.

This suggestion is reinforced by the course of events in the 69 patients $(40 \%)$ with signs as well as symptoms of local eye disease at the onset of their illness. It is most unlikely that these abnormal signs were secondary to blepharospasm. It also follows that these patients' initial symptoms were more likely to relate to their local eye disease than to a primary abnormality of the blink reflex. In 19 (11\%) patients, exemplified by Case 1 , the initial event was acute, painful, unilateral ocular pathology responding well to treatment. These patients usually blinked excessively as part of their presenting symptoms complex, and blepharospasm, worse on the affected side, developed within six months in 16 out of the 19. The close temporal relationships suggests that acute, painful focal eye or eye lid damage may have initiated the development of the dystonia in these patients. In the other 50 cases $(29 \%)$ there were chronic bilateral ocular abnormalities, usually blepharitis or keratoconjunctivities sicca. These tended to be resistant to local treatment and in the majority excessive blinking, which typically accompanies such chronic external eye disease, developed within six months of presentation. The evolution into blepharospasm, however, took considerably longer; over two years in 37 of the 50 cases (average 3.7 years). Thereafter dystonic movements frequently spread to involve the lower face, neck and other muscles. 
These data suggest that local eye disease may act as a stimulus to the development of blepharospasm. Existing theories of the pathophysiology of the condition invoke a disorder of blink reflex mechanisms ${ }^{4.5}$; the latencies of the blink and corneal reflexes are normal in established blepharospasm but the amplitude of both the $R_{1}$ and especially the $\mathrm{R}_{2}$ component of the blink reflex is increased, the duration of the $R_{2}$ and of the corneal reflex is prolonged, and the recovery of $R_{2}$ is abnormal. These findings suggest that the neuronal arcs of the reflexes are normal but that supranuclear influences (probably from the basal ganglia) increase the excitability of the involved brain stem interneurones (and motoneurones).

Two possibilities could explain how local ocular disease appears to trigger such abnormalities of the blink reflex in such a large proportion of the cases we have studied. (1) Either the local eye condition causes a change in the excitability of brain stem interneurones involved in the blink reflex, (2) those patients who develop blepharospasm in response to the stimulus of local ocular disease have a pre-existing abnormality of the brain stem interneurones which predisposes them to respond in this way. Some evidence would favour the second hypothesis. The vast majority of patients with local ocular disease of the sort documented in our patients do not develop blepharospasm. For instance the commonest local ocular condition found in our series was blepharitis, but we would estimate that less than 1 in 10,000 of those with blepharitis progress to blepharospasm. On the other hand blepharospasm was preceded by local ocular disease in eleven of the 14 patients $(79 \%)$ in our series who had first degree relatives with blepharospasm. This raises the possibility that a genetically determined susceptibility may be an important factor. We suggest therefore that the local ocular condition acts as a trigger to blepharospasm in those already predisposed, genetically or otherwise by an abnormality in brain stem interneuronal excitability, to develop the condition.

Such a sequence has also been suggested in other forms of focal dystonia. There is a surprisingly high incidence of local neck trauma preceding the development of spasmodic torticollis ${ }^{6}$, of dental work preceding isolated oro-mandibular dystonia ${ }^{7}$ and of local hand injury in those with dystonia writer's or musician's cramp ${ }^{8}$. The overall conclusion is that local injury or disease may trigger the dystonia in those with a pathophysiological substrate for this disease. In the case of blepharospasm the trigger is local eye disease in a substantial proportion of patients; the pathophysiological substrate is hyperexcitability of the brain stem interneurones involved in the blink reflex.

\section{References}

${ }^{1}$ Jankovic J. and Orman J: Blepharospasm : demographic and clinical survey of 250 patients. Ann Ophthalmol 1984, 16: 371-6.

${ }^{2}$ Marsden CD: Blepharospasm - oromandibular dystonia syndrome (Brueghel's Syndrome). $J$ Neurol, Neurosurg Psychiat 1976, 39: 1204-9.

${ }^{3}$ Henderson JW: Essential blepharospasm. Trans Am Ophthalmol Soc 1956, 54: 453-520.

${ }^{4}$ Berardelli A, Rothwell JC, Day BL, Marsden CD: Pathophysiology of blepharospasm and oromandibular dystonia. Brain 1985, 108: 593-608.

5 Jankovic J, Havins WE, Wilkins RB: Blinking and blepharospasm : mechanisms, diagnosis, and management. J Am Med Assoc 1982, 248: 316-3164.

${ }^{6}$ Sheehy MP and Marsden CD: Trauma and pain in spasmodic torticollis. Lancet 1980, i: 777-8.

${ }^{7}$ Koller WE: Edentulous orodykskinesia. Ann Neurol 1983, 13: 97-9.

${ }^{8}$ Sheehy MP and Marsden CD: Writer's cramp - a focal dystonia. Brain 1982, 105: 461-80. 